\title{
Rheological Properties of İnjectable Hyaluronic Acid Hydrogels for Soft Tissue Engineering Applications
}

\author{
Gizem Kaya ${ }^{1}$, Faruk Oytun ${ }^{1, *}$ \\ 1 VSY Biotechnology, R\&D Laboratories, Tuzla, Istanbul, 34959, Turkey \\ * Correspondence: foytun@ vsybiotechnology.com;
}

Scopus Author ID 55625860900

Received: 2.07.2020; Revised: 23.07.2020; Accepted: 24.07.2020; Published: 28.07.2020

\begin{abstract}
Hydrogels are cross-linked three-dimensional (3D) polymeric network, which can hold the water within its porous structure. They have recently been used in various biomedical applications. In this study, injectable hyaluronic acid (HA) hydrogels were prepared using different concentrations of 1,4-butanediol diglycidyl ether (BDDE) as a crosslinker (1-5\% w/w) and investigated their rheological, swelling and injectability properties. The results demonstrated that the rheological characteristics of hydrogels enhanced with increasing crosslinker concentration. The elastic modulus of the hydrogels ranged from $280 \mathrm{~Pa}$ to $990 \mathrm{~Pa}$, while the complex viscosities were found between $42 \mathrm{~Pa}$.s and $190 \mathrm{~Pa}$.s at an oscillation frequency of $1 \mathrm{~Hz}$. These results clearly suggest that the injectable HA hydrogels are a potential candidate for various soft tissue engineering applications due to their highly tunable rheological properties.
\end{abstract}

Keywords: Crosslinking; biomaterials; hydrogel; viscoelasticity; rheology; soft tissue engineering.

(C) 2020 by the authors. This article is an open-access article distributed under the terms and conditions of the Creative Commons Attribution (CC BY) license (https://creativecommons.org/licenses/by/4.0/).

\section{Introduction}

Hydrogels are promising three-dimensional (3D) polymeric networks which have outstanding characteristics such as high water uptake, good biocompatibility, and tunable biomechanical and viscoelastic properties [1-4]. They have been mostly used in many biomedical applications, including drug delivery, wound dressing, biosensors, prevention of postoperative adhesions, orthopedic or aesthetic injections, and contact lenses [5-12]. In particular, injectable hydrogels have considerable attracted interest in the biomedical industry, due to their low invasive surgical procedure, adhering to tissue and filling properties [13-16].

Hyaluronic acid (HA) is one of the most preferred biopolymers in injectable hydrogels, which consists of repeating D-glucuronic acid and $\mathrm{N}$-acetyl D-glucosamine disaccharide units [17-19]. It exhibits interesting viscoelastic and lubricating properties, excellent biocompatibility, and biodegradability [20]. In contrast to these advantages, its poor mechanical stability and rapid degradation limit its use in biomedical applications [21]. It is a known fact that the half-life of HA in the human body is less than one day [22]. Therefore, it cannot be expected to be long-lasting in injected areas. To overcome this drawback, many cross-linking agents such as 1,4-butanediol diglycidyl ether (BDDE), 1- ethyl-3-(3dimethylaminopropyl) carbodiimide (EDC), divinyl sulfone (DVS) and glutaraldehyde (GTA) have been used to improve its biomechanical properties [23-25]. Among them, BDDE is widely used in much dermal filler and intraarticular viscosupplements due to its low 
cytotoxicity and sensitization. It can easily react with hydroxyl groups to form ether bonds by the epoxide ring-opening of itself in an alkaline environment [26].

The crosslinker ratio is strongly associated with the degree of cross-linking. The crosslinking degree is increased with the increase of crosslinker amount resulting in highly stable and durable hydrogel against enzymatic degradation. Therefore, in this study, injectable HA hydrogels were prepared at different concentrations of BDDE, and the effect of crosslinker concentration on rheological properties was evaluated.

\section{Materials and Methods}

\subsection{Materials.}

Hyaluronic acid sodium salt (molecular weight $\left.=7.66 \times 10^{5} \mathrm{Da}\right)$ was purchased from Shiseido. 1,4-butanediol diglycidyl ether (BDDE) was provided from Sigma Aldrich. All other chemicals were of analytical grade and used as received without any purification.

\subsection{Preparation of HA hydrogels at different crosslinker concentrations.}

10 wt \% of HA sodium salt was first dissolved in $0.25 \mathrm{M}$ sodium hydroxide $(\mathrm{NaOH})$ solution by a mechanical stirrer in $300 \mathrm{rpm}$ for $30 \mathrm{~min}$. After the HA was completely dissolved, BDDE at different concentrations $(1,2,3$, and $5 \mathrm{wt} \%$ with respect to the total mixture) was added to HA solution and stirred for an additional $5 \mathrm{~min}$. The cross-link reaction was then performed at $45^{\circ} \mathrm{C}$ for 4 hours. The prepared hydrogels were named as HA1, HA2, HA3, and HA5, respectively (Figure 1). After the reaction, the hydrogels were put into deionized water (DIW) following by neutralization with $0.1 \mathrm{M}$ of hydrochloric acid $(\mathrm{HCl})$. The hydrogels were then repeatedly washed and swollen in DIW to remove unreacted substances. The resulting hydrogels were then precipitated in excess ethanol and dried under vacuum oven at room temperature for 24 hours. Finally, the hydrogels with a concentration of $20 \mathrm{mg} / \mathrm{ml}$ were filled into $1 \mathrm{~mL}$ syringes and autoclaved in air-stream at $121^{\circ} \mathrm{C}$ for $15 \mathrm{~min}$.

\subsection{Swelling Test.}

A certain amount of dried hydrogels was immersed into a physiological saline solution $(\mathrm{NaCl} 0.9 \% \mathrm{w} / \mathrm{w})$ for $24 \mathrm{~h}$ and then separated by filtration. The swelling ratio (SR) of the hydrogels was calculated using Eq. (1). Measurements were performed three times, and the averages were calculated:

$$
S R(g / g)=\frac{W_{w}}{W_{d}}
$$

wherein $\mathrm{W}_{\mathrm{w}}$ and $\mathrm{W}_{\mathrm{d}}$ represent swollen and dry weights of the hydrogels, respectively.

\subsection{Characterization.}

Rheological measurements were performed using an Anton Paar MCR 102 rheometer equipped with a parallel plate geometry, $25 \mathrm{~mm}$ plate diameter and $1.0 \mathrm{~mm}$ gap, while the injection force tests were carried out at a compression rate of $12 \mathrm{~mm} / \mathrm{min}$ by Lloyd-LS1 testing device. 


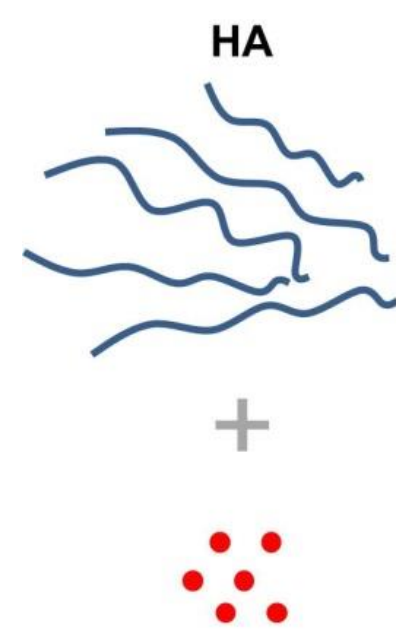

BDDE
HA1

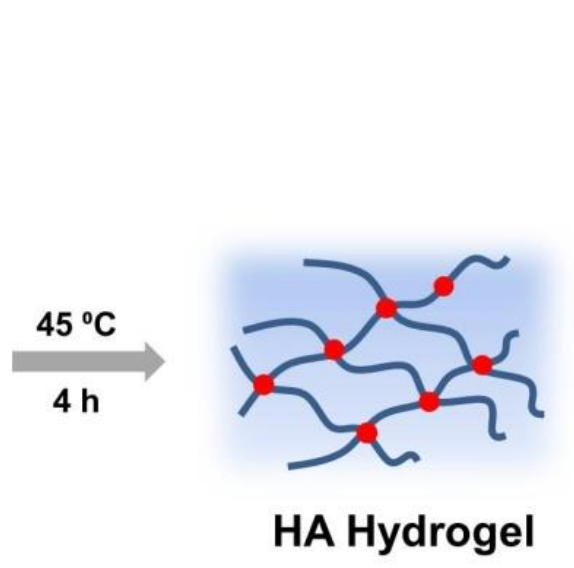

HA5

Soft Gel

HA2

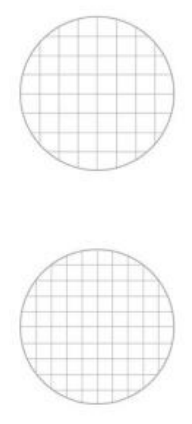

HA3

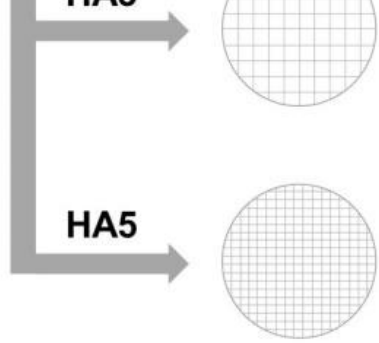

Hard Gel

Figure 1. Schematic illustration of HA hydrogels at different crosslinker concentration.

\section{Results and Discussion}

The swelling ability of the hydrogels is a crucial parameter because it characterizes their capacity to maintain the structure and retain sufficient water, affecting all characteristics of the hydrogel in relevant applications such as stiffness, degradation, and biocompatibility $[27,28]$. To understand the influence of the crosslinker concentration on the swelling behavior of the hydrogels, the swelling ratio was investigated by immersing the dried hydrogels in saline solution to reach equilibrium. As shown in Figure 2a, all hydrogels showed various swelling ratios, ranging from 42 to $194 \mathrm{~g} / \mathrm{g}$. The water uptake ability significantly decreased, as the crosslinker concentration increased from 1 to $5 \mathrm{wt} \%$. These findings indicated that the hydrogels prepared at different crosslinker concentrations might be dominant on swelling behavior.

Injectable hydrogels with different biomechanical properties possess different rheological characteristics such as viscosity, elasticity, and plasticity. The rheological properties of injectable hydrogels are clinically relevant because they play a critical role in identifying how the hydrogel behaves after injection. The most significant rheological parameters for injectable hydrogels are regarded as complex viscosity $\left(\eta^{*}\right)$ and elastic modulus $\left(G^{\prime}\right)$. Complex viscosity measures the ability of the gel to resist shearing forces within a tissue after injection, while elastic modulus measures the stiffness of hydrogel and its interactions [29]. These parameters determine how well it resists tension forces due to injected area movements after injection.

The elastic modulus of hydrogels as a function of the oscillation frequency are shown in Figure 2b. It can be noted that the elastic modulus of hydrogels increased with increasing crosslinker concentration. The elastic modulus of HA1, HA2, HA3, and HA5 were found as $280 \mathrm{~Pa}, 450 \mathrm{~Pa}, 680 \mathrm{~Pa}$, and $990 \mathrm{~Pa}$ at $1 \mathrm{~Hz}$, respectively (Figure 2c). The elastic modulus is especially important in determining the place of use in dermal filler applications. The high elastic modulus shows that the filler is firm and should be placed in deeper facial areas to reduce the tangibility in the dermis [30]. These types of highly resistant fillers can also be used 
to correct nasal bridges. The fillers with lower elastic modulus show more fluid character, and they can be used in fine lines, which is more sensitive and over larger areas like the cheeks or cheekbones. Consequently, the results exhibited that the rheological properties of HA hydrogels improved with increasing crosslinker concentration. In addition, it has been shown that hydrogels with different elastic modulus values can be used as dermal fillers in different areas of the face.
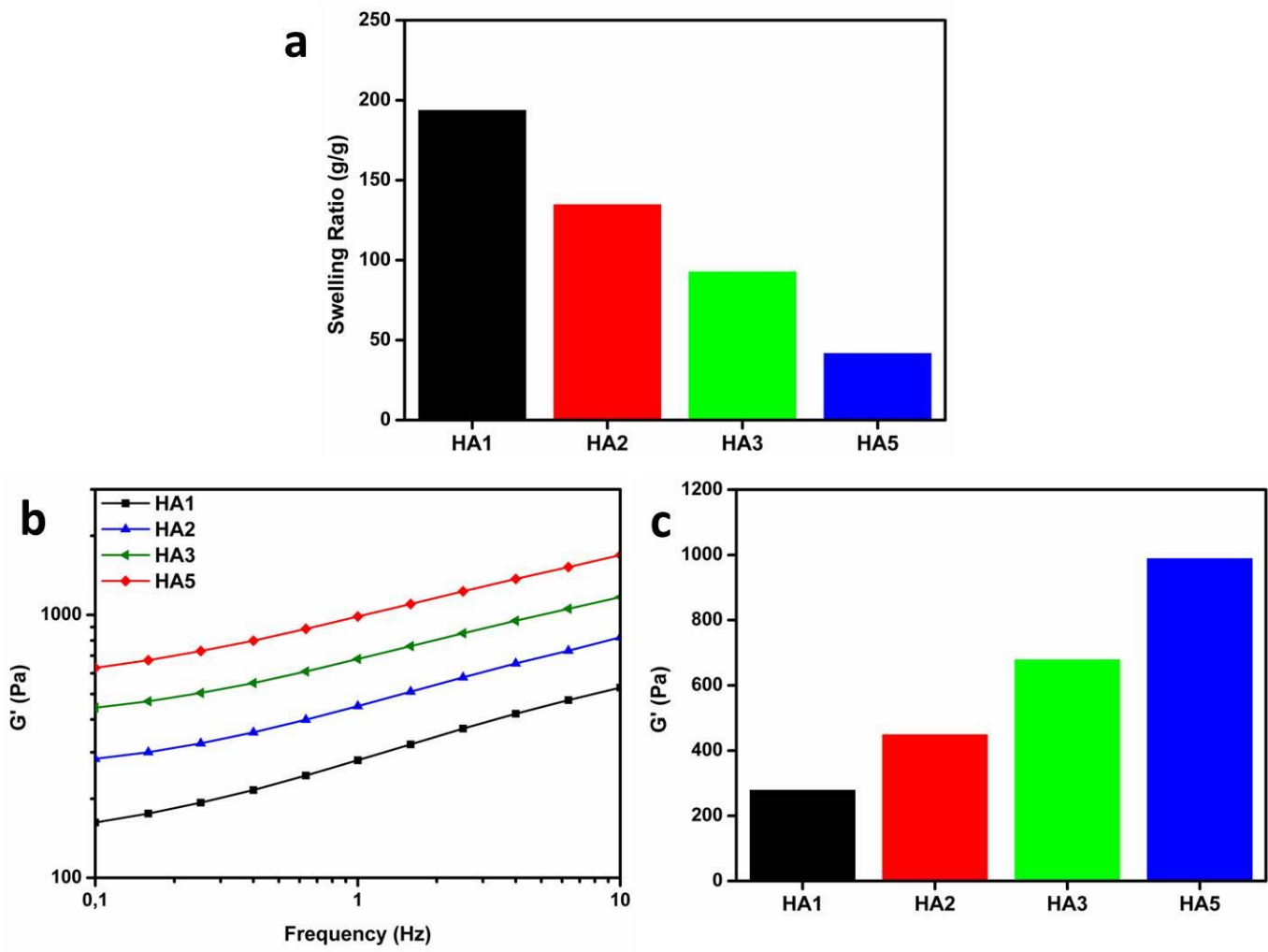

Figure 2. a) Swelling ratios of HA hydrogels, b) Elastic $\left(G^{\prime}\right)$ modulus of hydrogels as a function of the oscillation frequency, c) bar graph showing $G^{\prime}$ modulus for hydrogels.

On the other hand, the complex viscosity at $1 \mathrm{~Hz}$ of hydrogels was shown in Figure $3 \mathrm{a}$. The complex viscosity greatly decreased with increasing oscillation frequency, demonstrating that the hydrogels were highly shear thinning. The differences in complex viscosity can be related to the structural strength of hydrogels having different crosslinker concentrations. As shown in Figure $3 b$, hydrogels exhibited a higher viscosity with increasing crosslinker concentration. The complex viscosities were found as 42, 74, 107, and $190 \mathrm{~Pa}$.s for HA1, HA2, HA3, and HA5, respectively. This showed that the complex viscosity of the hydrogels was influenced by the crosslinker concentration, and the increase in the crosslinker content of the hydrogel resulted in high complex viscosity. Moreover, injection force tests were performed to demonstrate the injectability of hydrogels during application. As shown in Figure 3c, it was clear that the injection force increased from $10 \mathrm{~N}$ to $31 \mathrm{~N}$ when the crosslinker concentration increased from $1 \mathrm{wt} \%$ to $5 \mathrm{wt} \%$ in the hydrogel matrix. Indeed, the injection forces in this regime are proportional to the elastic modulus. This was related to the high viscosity of the hydrogels when a higher crosslinker concentration was introduced. The results demonstrated that the increase in crosslinker concentration increased the injection force. When considering all factors, the increase in crosslinker concentration improves the rheological properties of the hydrogels but also leads to the injection force. 

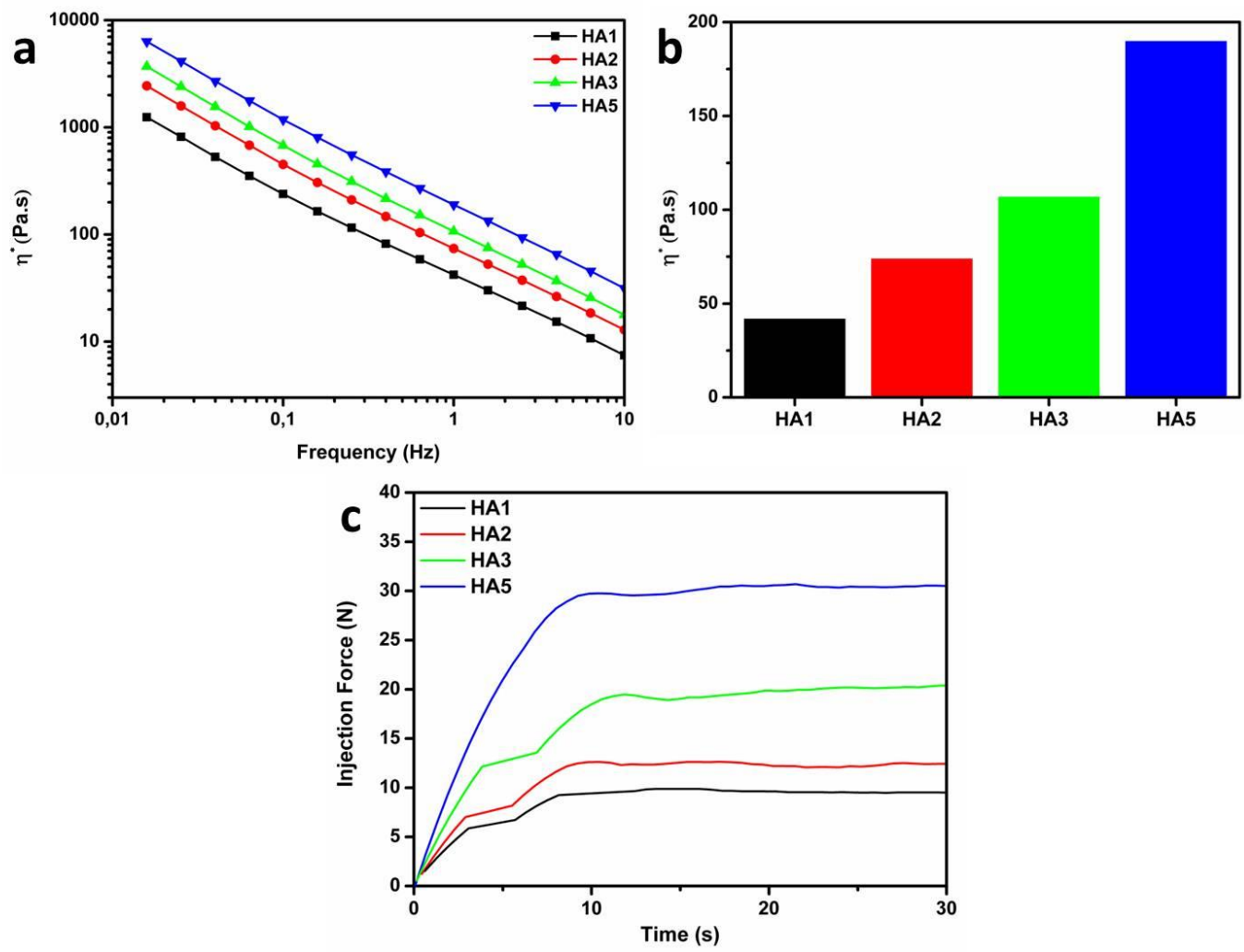

Figure 3. a) Complex viscosity $(\eta *)$ as a function of the oscillation frequency, b) bar graph showing complex viscosity for hydrogels, c) Graph showing extrusion force overtime for hydrogels injected from a $1 \mathrm{~mL}$ syringe with needles of $27 \mathrm{G}$.

\section{Conclusions}

In this study, HA hydrogels were prepared at different concentrations of BDDE, and the effect of crosslinker concentration on rheological properties was evaluated. With increasing crosslinker concentration in the hydrogel matrix, the swelling ability of hydrogels significantly decreased, while they exhibited high elastic modulus and complex viscosity. The rheological analyses showed that the elastic modulus and complex viscosity of hydrogels were strongly dependent on crosslinker concentration, offering a means of controlling the biomechanical properties for injectable applications. The results clearly suggest that the HA hydrogels are especially a superior candidate for injectable applications due to their highly tunable properties.

\section{Funding}

This study was financially supported by The Scientific and Technological Research Council of Turkey (TUBITAK) (Project No: 3180462).

\section{Acknowledgments}

This research has no acknowledgment.

\section{Conflicts of Interest}

The authors declare no conflict of interest,

\section{References}


1. Li, Q.; Liu, C.; Wen, J.; Wu, Y.; Shan, Y.; Liao, J. The design, mechanism and biomedical application of self-healing hydrogels. Chinese Chemical Letters 2017, 28, 1857-1874, https://doi.org/10.1016/j.cclet.2017.05.007.

2. Driest, P.J.; Dijkstra, D.J.; Stamatialis, D.; Grijpma, D.W. Tough combinatorial poly(urethane-isocyanurate) polymer networks and hydrogels synthesized by the trimerization of mixtures of NCO-prepolymers. Acta Biomaterialia 2020, 105, 87-96, https://doi.org/10.1016/j.actbio.2020.01.025.

3. Saunders, L.; Ma, P.X. Self-Healing Supramolecular Hydrogels for Tissue Engineering Applications. Macromolecular Bioscience 2019, 19, https://doi.org/10.1002/mabi.201800313.

4. $\quad$ Elkhoury, K.; Russell, C.S.; Sanchez-Gonzalez, L.; Mostafavi, A.; Williams, T.J.; Kahn, C.; Peppas, N.A.; Arab-Tehrany, E.; Tamayol, A. Soft-Nanoparticle Functionalization of Natural Hydrogels for Tissue Engineering Applications. Advanced Healthcare Materials 2019, 8, https://doi.org/10.1002/adhm.201900506.

5. Gritsch, L.; Motta, F.L.; Contessi Negrini, N.; Yahia, L.H.; Farè, S. Crosslinked gelatin hydrogels as carriers for controlled heparin release. Materials Letters 2018, 228, 375-378, https://doi.org/10.1016/j.matlet.2018.06.047.

6. Koehler, J.; Brandl, F.P.; Goepferich, A.M. Hydrogel wound dressings for bioactive treatment of acute and chronic wounds. European Polymer Journal 2018, https://doi.org/10.1016/j.eurpolymj.2017.12.046.

7. Jing, X.; Wang, X.-Y.; Mi, H.-Y.; Turng, L.-S. Stretchable gelatin/silver nanowires composite hydrogels for detecting human motion. Materials Letters 2019, 237, 53-56, https://doi.org/10.1016/j.matlet.2018.11.078.

8. Oh, S.H.; Kang, J.G.; Lee, J.H. Co-micellized Pluronic mixture with thermo-sensitivity and residence stability as an injectable tissue adhesion barrier hydrogel. Journal of Biomedical Materials Research Part B: Applied Biomaterials 2018, 106, 172-182, https://doi.org/10.1002/jbm.b.33824.

9. Hemmati-Sadeghi, S.; Dey, P.; Ringe, J.; Haag, R.; Sittinger, M.; Dehne, T. Biomimetic sulfated polyethylene glycol hydrogel inhibits proteoglycan loss and tumor necrosis factor- $\alpha$-induced expression pattern in an osteoarthritis in vitro model. Journal of Biomedical Materials Research Part B: Applied Biomaterials 2019, 107, 490-500, https://doi.org/10.1002/jbm.b.34139.

10. Korogiannaki, M.; Jones, L.; Sheardown, H. Impact of a Hyaluronic Acid-Grafted Layer on the Surface Properties of Model Silicone Hydrogel Contact Lenses. Langmuir 2019, 35, 950-961, https://doi.org/10.1021/acs.langmuir.8b01693.

11. Mao, X.; Cheng, R.; Zhang, H.; Bae, J.; Cheng, L.; Zhang, L.; Deng, L.; Cui, W.; Zhang, Y.; Santos, H.A.; Sun, X. Self-Healing and Injectable Hydrogel for Matching Skin Flap Regeneration. Advanced Science 2019, 6.

12. Prince, D.A.; Villamagna, I.J.; Borecki, A.; Beier, F.; de Bruyn, J.R.; Hurtig, M.; Gillies, E.R. Thermoresponsive and Covalently Cross-Linkable Hydrogels for Intra-Articular Drug Delivery. ACS Applied Bio Materials 2019, 2, 3498-3507, https://doi.org/10.1021/acsabm.9b00410.

13. Qu, J.; Zhao, X.; Liang, Y.; Xu, Y.; Ma, P.X.; Guo, B. Degradable conductive injectable hydrogels as novel antibacterial, anti-oxidant wound dressings for wound healing. Chemical Engineering Journal 2019, 362, 548-560, https://doi.org/10.1016/j.cej.2019.01.028.

14. Tan, H.; Marra, K.G. Injectable, Biodegradable Hydrogels for Tissue Engineering Applications. Materials 2010, 3, 1746-1767, https://doi.org/10.3390/ma3031746.

15. Sisso, A.M.; Boit, M.O.; DeForest, C.A. Self-healing injectable gelatin hydrogels for localized therapeutic cell delivery. Journal of Biomedical Materials Research Part A 2020, 108, 1112-1121, https://doi.org/10.1002/jbm.a.36886.

16. Pal, A.; Smith, C.I.; Palade, J.; Nagaraju, S.; Alarcon-Benedetto, B.A.; Kilbourne, J.; Rawls, A.; WilsonRawls, J.; Vernon, B.L.; Nikkhah, M. Poly(N-isopropylacrylamide)-based dual-crosslinking biohybrid injectable hydrogels for vascularization. Acta Biomaterialia 2020, 107, 138-151, https://doi.org/10.1016/j.actbio.2020.02.041.

17. Kwon, M.Y.; Wang, C.; Galarraga, J.H.; Puré, E.; Han, L.; Burdick, J.A. Influence of hyaluronic acid modification on CD44 binding towards the design of hydrogel biomaterials. Biomaterials 2019, 222, https://doi.org/10.1016/j.biomaterials.2019.119451.

18. Agarwal, G.; K. V, K.; Prasad, S.B.; Bhaduri, A.; Jayaraman, G. Biosynthesis of Hyaluronic acid polymer: Dissecting the role of sub structural elements of hyaluronan synthase. Scientific Reports 2019, 9, https://doi.org/10.1038/s41598-019-48878-8.

19. Zamboni, F.; Ryan, E.; Culebras, M.; Collins, M.N. Labile cross-linked hyaluronic acid via urethane formation using bis( $\beta$-isocyanatoethyl) disulphide with tuneable physicochemical and immunomodulatory properties. Carbohydrate Polymers 2020, 245, https://doi.org/10.1016/j.carbpol.2020.116501.

20. Cao, W.; Sui, J.; Ma, M.; Xu, Y.; Lin, W.; Chen, Y.; Man, Y.; Sun, Y.; Fan, Y.; Zhang, X. The preparation and biocompatible evaluation of injectable dual cross-linking hyaluronic acid hydrogels as cytoprotective agents. Journal of Materials Chemistry B 2019, 7, 4413-4423, https://doi.org/10.1039/c9tb00839j.

21. Rao, N.V. Hyaluronic acid-based hydrogels for tissue engineering. In: Biomater. Organ Tissue Regen. 2020; pp. 551-565, https://doi.org/10.1016/b978-0-08-102906-0.00014-3.

22. Brown, T.J.; Laurent, U.B.; Fraser, J.R. Turnover of hyaluronan in synovial joints: elimination of labelled 
hyaluronan from the knee joint of the rabbit. Experimental Physiology 1991, 76, 125-134, https://doi.org/10.1113/expphysiol.1991.sp003474.

23. Khunmanee, S.; Jeong, Y.; Park, H. Crosslinking method of hyaluronic-based hydrogel for biomedical applications. Journal of Tissue Engineering 2017, 8, 1-16, https://doi.org/10.1177/2041731417726464.

24. McManamon, C.; Cameron, A.; de Silva, J.P.; Daly, R.; O'Brien, F.J.; Cross, G.L.W. Effect of cross-linking and hydration on microscale flat punch indentation contact to collagen-hyaluronic acid films in the viscoelastic limit. Acta Biomaterialia 2020, 111, 279-289, https://doi.org/10.1016/j.actbio.2020.04.043.

25. Xue, Y.; Chen, H.; Xu, C.; Yu, D.; Xu, H.; Hu, Y. Synthesis of hyaluronic acid hydrogels by cross-linking the mixture of high-molecular-weight hyaluronic acid and low-molecular-weight hyaluronic acid with 1,4butanediol diglycidyl ether. RSC Advances 2020, 10, 7206-7213, https://doi.org/10.1039/c9ra09271d.

26. De Boulle, K.; Glogau, R.; Kono, T.; Nathan, M.; Tezel, A.; Roca-Martinez, J.-X.; Paliwal, S.; Stroumpoulis, D. A Review of the Metabolism of 1,4-Butanediol Diglycidyl Ether-Crosslinked Hyaluronic Acid Dermal Fillers. Dermatologic Surgery 2013, 39, 1758-1766, https://doi.org/10.1111/dsu.12301.

27. Fan, L.; Yang, H.; Yang, J.; Peng, M.; Hu, J. Preparation and characterization of chitosan/gelatin/PVA hydrogel for wound dressings. Carbohydrate Polymers 2016, 146, 427-434, https://doi.org/10.1016/j.carbpol.2016.03.002.

28. Toledo, L.; Racine, L.; Pérez, V.; Henríquez, J.P.; Auzely-Velty, R.; Urbano, B.F. Physical nanocomposite hydrogels filled with low concentrations of $\mathrm{TiO} 2$ nanoparticles: Swelling, networks parameters and cell retention studies. Materials Science and Engineering: $C$ 2018, 92, 769-778, https://doi.org/10.1016/j.msec.2018.07.024.

29. Sundaram, H.; Voigts, B.O.B.; Beer, K.; Meland, M. Comparison of the Rheological Properties of Viscosity and Elasticity in Two Categories of Soft Tissue Fillers: Calcium Hydroxylapatite and Hyaluronic Acid. Dermatologic Surgery 2010, 36, 1859-1865, https://doi.org/10.1111/j.1524-4725.2010.01743.x.

30. Rohrich, R.J.; Bartlett, E.L.; Dayan, E. Practical Approach and Safety of Hyaluronic Acid Fillers. Plastic and Reconstructive Surgery - Global Open 2019, 7, https://doi.org/10.1097/gox.0000000000002172. 